\title{
A New Method of Uplink Power Compensation of Rain Attenuation of Satellite Communication System
}

\author{
Li Rong \\ College of Communication and Information Engineering, Xi'an University of Science and \\ Technology, Xi'an 710054, China \\ Cold3000@126.com
}

Key words: Satellite communication; Reduction of rain attenuation; Diversity reception;

\begin{abstract}
In satellite communication the signal fading may reach the maximum in rain weather. In this paper, an uplink power reduction rain fading method is put forward to overcome the shortcoming of the traditional method. Through detection of satellite signal strength, the rain attenuation in satellite communication link can be calculated, and according to the rain attenuation values, ground station power will be adjusted to achieve the maximum compensation for the rain attenuation, effectively reducing the influence of rain attenuation on the communication quality.
\end{abstract}

\section{Introduction}

With the widespread application and high speed development of modern communications, the demand of people for modern communications is becoming more anxious. Satellite communication has become one of the most important technologies in recent years because of its features such as wide coverage, no geographical limitations, cost being nothing to do with distance ${ }^{[1]}$. The technology of satellite communication resolves the problems of traditional ground communication systems which are restricted to geographical surrounding and economic conditions, providing global seamless full coverage networks. However, because of space radio transmission satellite commutation is highly susceptible to cloud, snow, fog and rain, which leads to various degree rain fading. The research indicates that the fading will reach the maximum in rainy weather. Consequently, how to decrease the attenuation and improve the communication quality have become the hot issues.

However, the traditional diversity reception technology is to choose two sites with certain distance by using the space heterogeneity in rain areas and two earth stations are located. The two stations that have certain distance receive the signals of satellite communication respectively. Then, the rain attenuation values of the signals are calculated. By comparison, the signal which has less attenuation is selected as the ultimate reception signal. The method may reduce the influence of rain fading, but it cannot effectively remove the influence of rain attenuation.

Based on above problems an uplink power adjustment method is put forward. Through detection of satellite signal strength, the rain attenuation in satellite communication link can be calculated, and according to the rain attenuation values, ground station power will be adjusted to achieve the maximum compensation for the rain attenuation, effectively reducing the influence of rain attenuation on the communication quality.

\section{The Influence of rain fading on satellite communication}

Satellite communication is the technology that can keep two or more earth stations communicate with each other through the relay of the manual communication satellite. ${ }^{[3]}$ The radio signals spread between the ground stations and the satellite, travelling through the atmosphere. The signals, while spreading in the atmosphere, are inevitably affected by the energy reflection and absorption of ions and free electrons in the atmosphere. Moreover, the troposphere has large amount of water vapor molecules, CO molecules, O molecules, and various aerosols such as snow, fog, and rain. Thus, the radio signals, while spreading in the troposphere, are continually absorbed, reflected and scattered, causing the energy reduction. 
Among the attenuation, the rain fading for the signals is the most serious. In rainy days, the electromagnetic waves of satellite communication are absorbed or reflected by the rain drops, causing the attenuation. The influence extent is determined by the microscopic characteristics of the rain drops. The signal attenuation values with different powers are different, affected by different rainfall intensity. In general, the rain attenuation of signals becomes particularly serious when the power exceeds $10 \mathrm{~Hz}^{[2]}$. Fig. 1 shows the relationship between the signal power and rain attenuation.

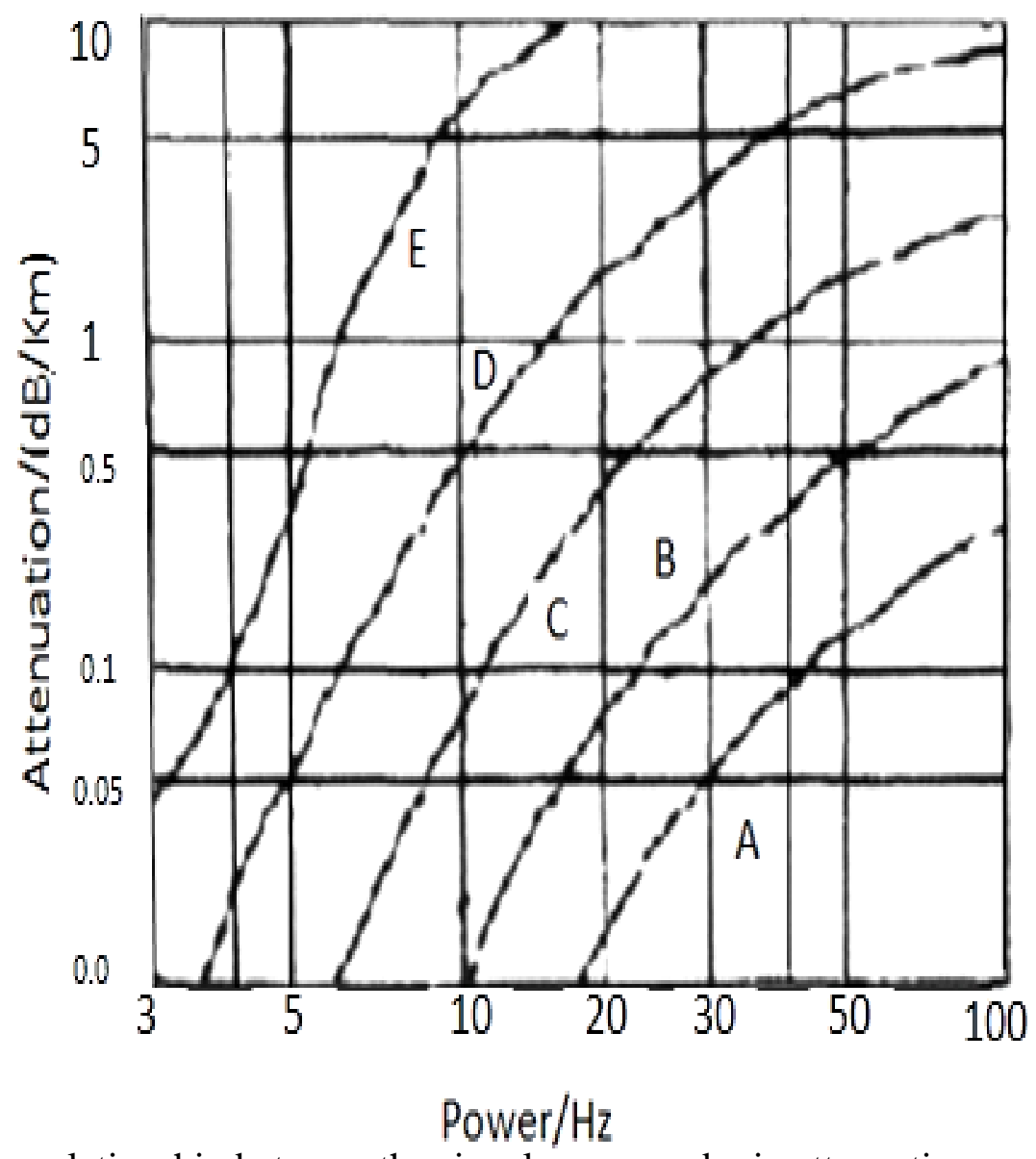

Fig.1. the relationship between the signal power and rain attenuation

Fig.1 indicates that the attenuation values of the radio signals are different with the different rainfall intensity. The traditional diversity reception, as stated above, is that two earth stations receive the radio signals reflected by the satellite simultaneously and the respective rain attenuation of two stations is different because of the different rainfall intensity. Then calculating the attenuation degrees of the reception signals, the equation is as follows

$$
A_{r}(d B)=k R_{P}^{b} \bullet L_{e}
$$

where $\boldsymbol{R}_{P}$ represents the rate of rainfall, b is the coefficient of the rainfall rate and $\boldsymbol{L}_{e}$ is the transmission path of the signals in the rain areas. Thus, the respective rain attenuation of the two stations is calculated and by comparison, the ultimate reception signals is selected which has less rain attenuation. The equation of the quality of satellite communication is as Eq. 2

$$
\alpha=\frac{R \bullet L_{e}}{A_{r}} \bullet \frac{\log P-b}{R_{P}}
$$

As Eq.2 states, the quality of satellite communication is inversely proportional to the rain attenuation. It indicates that the less the signal is affected by rain fading, the better the quality is. Though the signal with the less rain attenuation is selected to reduce the influence of rain by traditional diversity reception, the attenuation cannot be removed affectively, causing the reduction of communication quality.

Therefore, in order to overcome the influence of rain fading on communication signals and the shortcoming of the traditional technology, the method of uplink power control is put forward. 
Through detection of satellite signal strength, the rain attenuation in satellite communication link can be calculated, and according to the rain attenuation values, ground station power will be adjusted to achieve the maximum compensation for the rain attenuation, effectively reducing the influence of rain attenuation on the communication quality.

\section{The Method of Uplink Power Compensation of rain attenuation}

The method of traditional diversity reception to reduce rain attenuation is to select the signal which is affected less by rain attenuation and it cannot avoid the influence of rain fading absolutely. So we put forward the method of uplink power control to completely remove the rain attenuation, receiving high quality signals. The method of uplink power control to reduce rain fading will be introduced as follows.

\section{the Fundamental Link Principles}

Through the data of the uplink and according to the link rain attenuation values calculated by communication data, the ground station emission power can be adjusted by the influence value of rain fading. Then, to increase the transmitting power accordingly counteracts the power loss caused by rainfall and the same signals as that in normal weathers can be received by the ground station to reduce the rain attenuation.

The specific principle is as follows: as the uplink rain fading values cannot be calculated directly, the downlink rain fading values that can be measured directly are used to get the uplink power estimated values by frequency conversion. Then, the estimated values are sent to the system processor to achieve the compensation for the rain attenuation by power control. The block diagram is shown in Fig. $2^{[4]}$

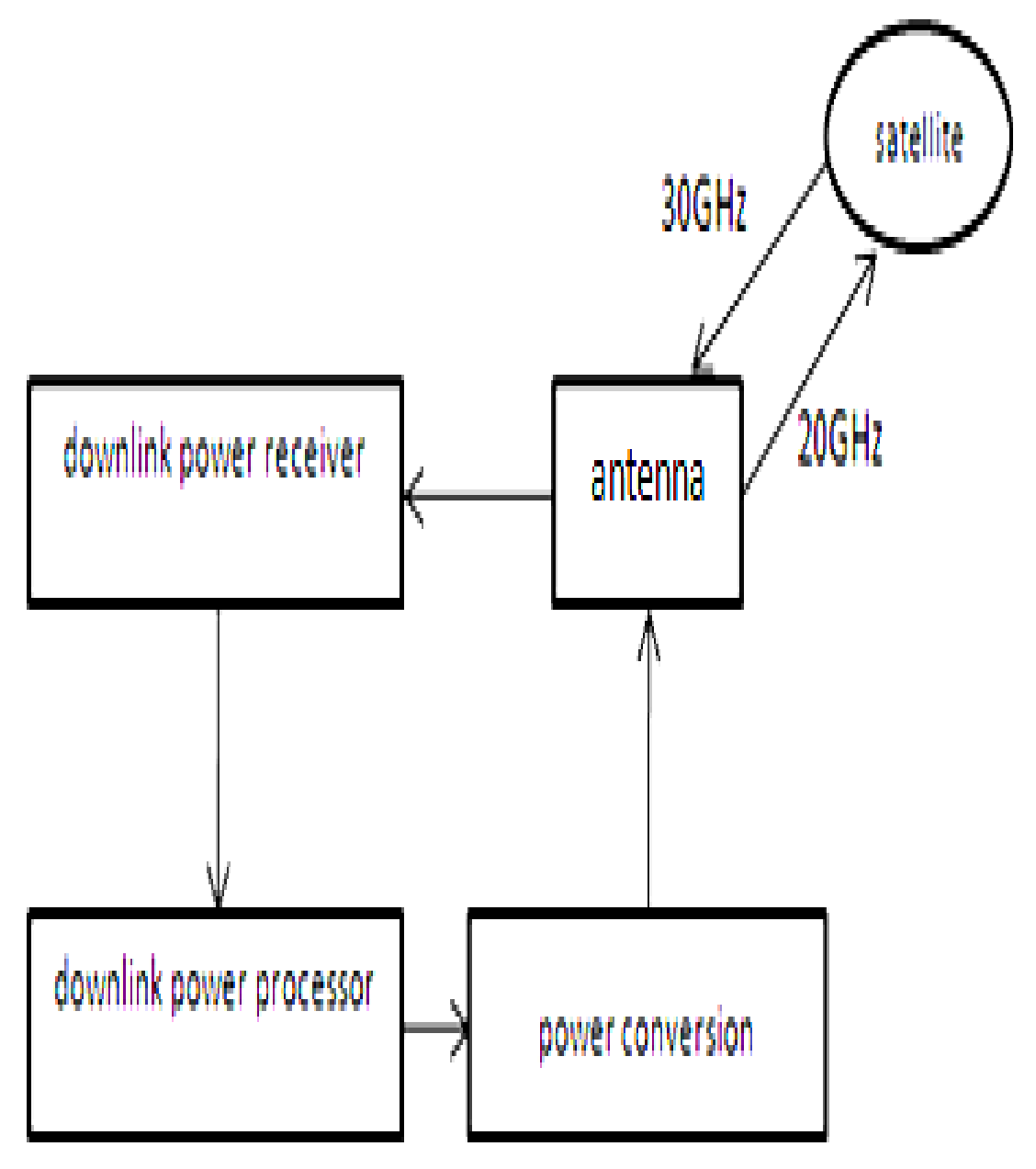

Fig 2. The priciple block diagram of uplink power control 


\section{the Estimation and Transformation of the Link Rain Fading}

As the real-time link attenuation cannot be measured directly, the attenuation value is predicted according to the experience values. The prediction equation based on the rain attenuation is as Eq.3

$$
\hat{A}_{30}(t / t-k T)=f A_{30}(t / t-T)+b A_{20}(t-k T)+e(t)
$$

where $e(t)$ is the prediction error value and T is the prediction intervals. In addition, $A_{30}(t)$ is the real value of the rain fading and $A_{30}(t-T)$ represents the real value which was measured at a time before this prediction. Thus, the prediction formula can be transformed to get the real rain fading equation as Eq.4

$$
A_{30}(t / t-k T)=b q^{-k} \sum_{i=1}^{\infty} f^{i} A_{20}(t-i T)=\frac{q^{-k} b}{1-f q^{-k}} A_{20}(t)
$$

where $k$ is the prediction delay number and $\boldsymbol{q}^{-1}$ is the delay factor. Moreover, the proportional factor will be $\frac{b}{1-r}$ when $q^{-1}$ is equal to 1. The Eq.4 can be simplified to Eq.5.

$$
\hat{A}_{30}(t / t-k T)=\frac{b}{1-f} A_{20}(t)
$$

now the prediction of the signal power is completed.

After the prediction of the downlink signal power, we will transform the downlink power to the uplink power. According to the rain attenuation of the downlink power signals, the uplink rain attenuation can be estimated by employing different frequencies. The equations of uplink power rain fading are as follows:

$$
\begin{aligned}
\frac{A_{u R}}{A_{d R}} & =\frac{\varphi\left(f_{u}\right)}{\varphi\left(f_{d}\right)} \\
\varphi(f) & =\frac{f^{1.72}}{1+3 \times 10^{-7} f^{3.44}}
\end{aligned}
$$

where $f$ represents the emission power of the signal and $A_{u R}(d B)$ and $A_{d R}(d B)$ indicate the uplink rain attenuation and the downlink rain attenuation respectively. Moreover, $f_{u}$ and $f_{d}$ are the uplink power and downlink power respectively.

Now the $A_{u R}$ can be calculated. Thus, the equation of the whole attenuation value is Eq.8

$$
L_{u}=A_{u R}+S_{u}
$$

where $L_{u}$ is the whole attenuation value. $A_{u R}$ is called the uplink rain attenuation and $S_{u}$ is the power attenuation when the signal travels in the atmosphere.

\section{the Compensation of the rain fading}

Now the whole power attenuation is calculated as above, so the emission power of the ground station may be adjusted according to the attenuation value, adding the attenuation value to the emission power. Eq.9 is the power calculation equation.

$$
P_{f}=P_{0}+L_{u}
$$

where $\boldsymbol{P}_{\boldsymbol{f}}$ is the ultimate signal power of the earth station and $\boldsymbol{P}_{\mathrm{o}}$ is the power which is existed under normal sunny weather conditions. Thus, the compensation for the rain attenuation will be finished in term of the Eq.9, effectively reducing the influence of rain attenuation on the communication quality.

\section{Conclusions}

The signal quality in satellite communication system will be affected by rain weather, so the rain attenuation would be overcome by using the compensation methods. In the paper, a new method based on uplink power compensation for rain attenuation is put forward. According to the calculation of the uplink rain fading by using the downlink rain fading, the ground station emission 
power can be adjusted to achieve the maximum compensation for rain attenuation. In a word, the new method overcomes the shortcoming of the traditional diversity reception and had high practical value for base-band digital signals. We will further study on the fields of application and carrier frequency estimation hereafter.

\section{References}

[1] Yao Jun, Li Baiping. Digital Microwave and Satellite Communication[M](In Chinese). Beijing University of Post and Telecommunications press, 2011,4

[2] Li Rong. Research of rain Attenuation of Ka-band Satellite Communication System [J]. ICNCE2013, 2013,8: 1526-1529

[3]Sun Xuekang, Zhang Zheng. Microwave and Satellite Communication [M](In Chinese). Post and Telecommunications press, 2003,6

[4] Zhao Zhenwen, Lu Changsheng. Prediction model of rain attenuation for the earth-space links[J] (In Chinese). Dianbo Kexue Xuebao/Chinese Journal of Radio Science, v 28, n 3, p 412-419, June 2013

[5] Badron K, Ismail A.F. Evaluation of RazakSAT's S-band link signal measurement with the radar derived rain attenuation. International Conference on Space Science and Communication, IconSpace, p 380-384, 2013

[6] Liu Guoliang. Satellite Communication[M](In Chinese). Xidian University Press, 2001

[7] Olurotimi E.O. Ojo J.S. Testing Rain Fall Models for Rain Ateenuation Prediction Purposes in Tropical Climate[J]. 31th URSI General Assembly and Scientific Symposium, p 1-4, 2014

[8] Kamruzzaman M. Islam M.S. Rain Attenuation Precdiction for Sattelite Communications Link at Ku and Ka Bands over Bangladesh[J]. ICEEICT 2014: p1-6.

[9] Livierators Spiros N. Kourogiorgas Charilaos I. On the Prediction of Joint Rain Attenuation Statistics in Earth-space Diversity Systems using Copulas[J]. IEEE Transactions on Antennas and Propagation,V62, 2250-2257,2014.

[10]Yussuff Abauoni Isiaka. Khamis NorHisham Haji. Rain Attenuation Prediction Model for Lagos at Millimeter Wave Band [J]. Journal of Atmospheric and Oceanic Technology,V31, p 639-646,2014

[11]Garcia Jose M. Riera Jose M. Rain attenuation variability calculated from a slant-path Ka-band experiment[J]. 8th European Conference on Antennas and Propagation, p 676-679,2014.

[12]Abdulrahman A,Y. Rahman T.A. Investigation of the Unified Rain Attenuation Prediction Method with Data from Tropical Climates[J]. IEEE Antennas and Wireless Propagation Letters, v 13, p 1108-1111, 2014.

[13]Maekama Yasuyuki, Inamori Yutaka. Effects of Rain Area Motions and Ground Wind Velocities on Rain Attenuation Characteristics of Ku-band Satellite Signals[J]. URSI GASS 2014.

[14]Kalogiros John, Anagnostou Marious N. Evaluation of a New Polar metric Algorithm for Rain-path Attenuation Correction of x-band Radar Observations Ggainst Disdrometer[J]. IEEE Transactions on Geoscience and Remote Sensing, p 1369-1380,2014.

[15]Poolsawut Jirutchaya, Linpiti Tulaya. A Short-term Rain-induced Attenuation Model for Satellite Link Quality Prediction[J]. ECTI-CON 2014.

[16]Sun Jiejing, Guo Lixin, Ge Jianhua. Effects of Rain Attenuation on Ray-tracing Prediction in Urban Microcellular Environment at $20 \mathrm{GHz}[\mathrm{J}]$.

2014 IEEE International Wireless Symposium, p 234-237, 2014. 